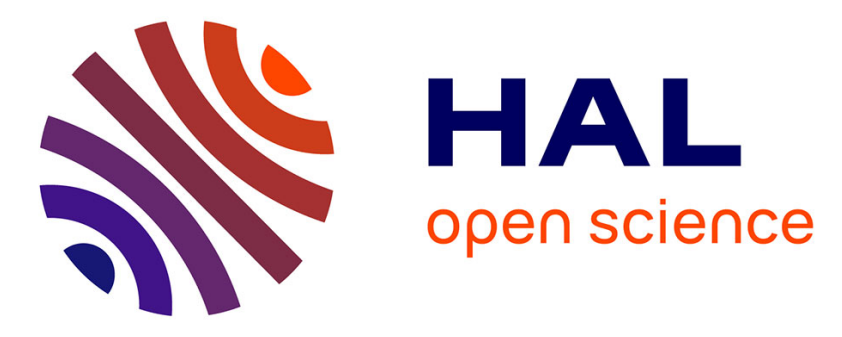

\title{
3D Interaction Assistance Through Context-Awareness: A semantic reasoning engine for classic virtual environment
}

\author{
Yannick Dennemont, Guillaume Bouyer, Samir Otmane, Malik Mallem
}

\section{- To cite this version:}

Yannick Dennemont, Guillaume Bouyer, Samir Otmane, Malik Mallem. 3D Interaction Assistance Through Context-Awareness: A semantic reasoning engine for classic virtual environment. International Conference on Computer Graphics Theory and Applications (GRAPP 2012), Feb 2012, Roma, Italy. pp.562-567, 10.1109/VR.2012.6180903 . hal-00672645

\section{HAL Id: hal-00672645 \\ https://hal.science/hal-00672645}

Submitted on 21 Feb 2012

HAL is a multi-disciplinary open access archive for the deposit and dissemination of scientific research documents, whether they are published or not. The documents may come from teaching and research institutions in France or abroad, or from public or private research centers.
L'archive ouverte pluridisciplinaire HAL, est destinée au dépôt et à la diffusion de documents scientifiques de niveau recherche, publiés ou non, émanant des établissements d'enseignement et de recherche français ou étrangers, des laboratoires publics ou privés. 


\title{
3D INTERACTION ASSISTANCE THROUGH CONTEXT-AWARENESS A semantic reasoning engine for classic virtual environment
}

\author{
Dennemont Yannick ${ }^{1}$, Guillaume Bouyer ${ }^{1}$, Samir Otmane ${ }^{1}$ and Malik Mallem ${ }^{1}$ \\ ${ }^{1}$ IBISC Laboratory, Evry University, 40 rue du pelvoux, 91020 Courcouronnes, France \\ FirstName.LastName@ibisc.univ-evry.fr
}

\begin{abstract}
Keywords: Interaction Techniques, Virtual reality, Context-awareness, Intelligent systems, Knowledge Representations
Abstract: $\quad$ This work focuses on 3D interaction assistance by adding adaptivity depending on the tasks, the objectives, and the general interaction context. The context is modelled with Conceptual Graphs (CG) based on an ontology. Including CG in our scene manager (Virtools) allows us to add semantic information and to describe the available tools. Adaptation result from rules handled with a logic programming layer (Prolog+CG) included in the Amine platform. This project is a step towards Intelligent Virtual Environments, which proposes a hybrid solution by adding a separate semantic reasoning to classic environments. The first experiment automatically manages few modalities depending on the distance to objects, user movement, available tools and modalities.
\end{abstract}

\section{Introduction}

3D interaction (3DI) immersion and usability are reinforced by the use of natural schemes. Meantime, adaptivity based on context is natural in human communication. Thus, we are designing a context and decision manager that focuses on expressiveness and usability in order to add adaptivity to 3DI. Through exploiting the context, we plan to best determine means to effectively help the user. We start by introducing some interests to achieve adaptative 3DI in the section 2 , then by positioning our work in the section 3 . We present in the section 4 a quick survey of methods for modelling and reasoning that motivates our approach. Afterwards sections 5 and 6 present our work in progress and perspectives.

\section{3D interaction adaptation interests}

3DI adaptation is an approach suggested by many researches (Wingrave et al., 2002)(Celentano and Nodari, 2004)(Bowman et al., 2006)(Octavia et al., 2010). As the "perfect" 3D interaction technique has yet to be found, a solution can be to adapt the interaction to a task, a device, etc., by adding specificity. Flavors, i.e known techniques variations, to improve usability are another form of adaptation. Applying automatically those kind of adaptations when needed defines an adaptative 3DI (see (Paramythis, 2009) for details about automatic adaptations types). Adaptative 3DI can be implicit with adaptations embedded in the interaction techniques (Poupyrev et al., 1996)(Boudoin et al., 2008), or explicit by using external processes (Lee et al., 2004)(Celentano and Nodari, 2004)(Bouyer et al., 2007)(Octavia et al., 2010). 3DI adaptation is an open issue which some advantages are:

- To speed up the interaction (Celentano and Nodari, 2004);

- To diminish the cognitive load (as in ubiquitous computing );

- To tailor the interaction (Wingrave et al., 2002) (Octavia et al., 2010);

- To add or manage interaction possibilities (Bouyer et al., 2007).

To achieve such adaptations, we can:

- Choose other techniques (Octavia et al., 2010) as the specificity idea;

- Make techniques variations (Octavia et al., 2010) as the flavor idea;

- Add/manage modalities (Irawati et al., 2005)(Bouyer et al., 2007)(Octavia et al., 2010);

- Perform automatically parts of the task (Celentano and Nodari, 2004). 


\section{Towards context-awareness}

In order to go beyond basic interaction, adaptive systems can first provide recognition of higher level information from raw data (on an activity recognition layer, Figure 1). But to achieve a better adaptivity, we need more content description: the context.

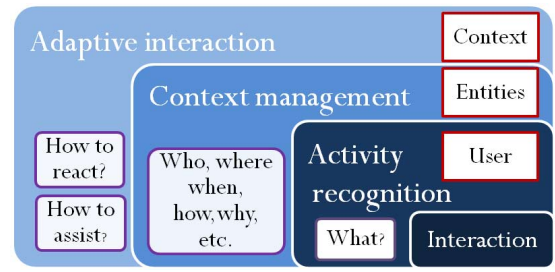

Figure 1: Different layers to reach adaptive interaction

A context formal and well recognized definition is (Dey and Abowd, 2000): Context is any information that can be used to characterize the situation of an entity. An entity is a person, place, or object that is considered relevant to the interaction between a user and an application, including the user and applications themselves. Thus, our system for 3DI is contextaware as it uses context to provide relevant information and/or services to the user, where relevancy depends on the user's task.

Context-awareness is a multidisciplinary field that uses similar tools than the knowledge representation and reasoning field. But it also requires computer science, physical sensors, cognitive sciences, etc. Intelligent systems have evolved (Brézillon, 2011) toward context-awareness and some encountered drawbacks can be explained by full abstract reasoning or user exclusion. Intelligent assistance systems can be split in two trend. Systems tend to stress user assistance on well defined context, e.g. (Bouyer et al., 2007), or to stress context identification that leads to direct adaptations for each situation, e.g. (Coppola et al., 2009). Context-aware applications have different main focuses (Figure 2) although they share an ideal list of properties to handle (Bettini et al., 2010):

- Heterogeneity and mobility of context;

- Relationships and dependencies between context;

- Timeliness: access to past and future states;

- Imperfection: data can be uncertain or incorrect;

- Reasoning: to decide or to derive information;

- Usability of modelling formalisms;

- Efficient context provisioning.

Ubiquitous computing tries to improve and soften the use of our surrounding chips and computers. Main focuses are communication between devices, possibilities of erroneous measurements and platforms limitations. Applications are numerous, like MoBe (Coppola et al., 2009) on smart phones.

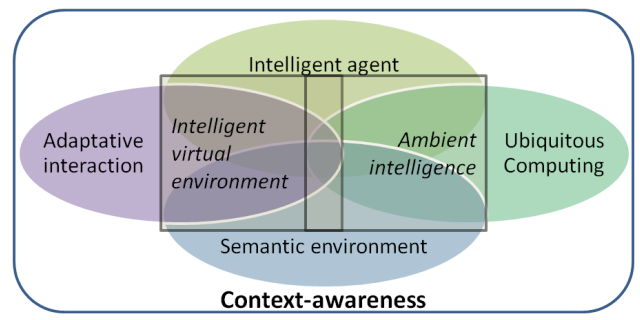

Figure 2: Different families of context-aware applications

Intelligent agents can be embodied in physical world (e.g. robot), virtual world (e.g. autonomous character) or can be hidden (e.g. genius loci (Celentano and Nodari, 2004)). Main features are communication issues, agent memory, ability to manage and reason on a local context, credibility or ability to reproduce a human behaviour.

Semantic environment stores its own description (e.g. the semantic web). Main focuses are: making environment comprehensive by machine (Peters and Shrobe, 2003), dealing with large scale environment, serving as a base for intelligence, automatic world construction (Bonis et al., 2008) or introducing semantic on the rendering loop core (Lugrin and Cavazza, 2007)(Soto et al., 1997).

Adaptative interaction tries to assist the user in his interaction with a virtual environment. A very specific localisation of adaptation like Go-Go (Poupyrev et al., 1996) or Fly-over (Boudoin et al., 2008) techniques (where interaction depends on the position of the user's hand) are good adaptative 3DI examples.

Intelligent Virtual Environments (Aylett and Luck, 2000) mix artificial intelligence, artificial life and virtual reality. It overlaps all classes and emphasises their reasoning capabilities. Applications often process semantic information from the environment (Celentano and Nodari, 2004)(Lugrin and Cavazza, 2007)(Latoschik et al., 2005)(Soto et al., 1997).

Ambient intelligence (Preuveneers, 2009) is a multidisciplinary area based on ubiquitous computing, artificial intelligence and semantic objects. It is basically the Intelligent Virtual Environments physical world version. Both overlap: e.g. intelligent physical sensors used in virtual reality, intelligent environments displayed on smart phones or mixed reality.

Our research is mainly in the adaptive 3D interaction field. Yet, to achieve wider and better 3D interaction, a richer context provided by adding or extracting semantic information in the environment and/or intelligent agents is needed. And reasoning needs will grow with the available information. So our approach is more generally a part of Intelligent Virtual Environment. Next, we discuss our choices for modelling context and reasoning to achieve these goals. 


\section{Knowledge Representation and Reasoning}

We need to manage context and to decide how to react, which is a form of Knowledge Representation and Reasoning (KR \& R). More precisely, our system needs first to retrieve and represent items of information, possibly specific to an application, then to handle this context and to define its effect on 3DI (discussed by (Frees, 2010) for virtual reality).

However representation and reasoning are not totally independent (e.g fuzzy logic reasoning needs a membership function). Besides, expressive representations are usually less reasoning efficient. Expressiveness is a measurement of the complexity of the expressible ideas, regardless of ease. Ease of expression and readability are included in usability.

Representations are various. Key-values are efficient but do not have real semantic. Markup-models typically introduce a fixed structure that brings semantic as an information hierarchy. Further, ontologies are definitions of concepts and relations that are interrelated. Graphical models like Unified or Context Modelling Language (UML and CML) and semantic networks have different level of expressiveness and efficiency but tend to usability. Conceptual Graphs (CG) are semantic networks interpreted on an ontology and achieve a better expressiveness.

For reasoning in bi-valued logic, ontology representation often uses Description logic (DL). First Order Logic (FOL) is more expressive but only semidecidable. Higher Order Logic (HOL) is interesting (some HOL functions are implemented in FOL engine) but has not an effective, sound and complete proof theory with standard semantic. Handling imperfection is useful. Multi-valued logic (like threevalued in CML) introduces alternatives from true or false. Further, fuzzy logic allows a simultaneous classification quantification with membership functions. Probability and probabilistic models like Bayesian Networks (BN) or Hidden Markov Models (HMM) quantify several hypotheses. Belief theory offers more quantification distribution possibilities. Neural Networks are deterministic but can also handle imperfection. As HMM, BN and machine learning methods, they classify situations without explicit semantic since trained on examples' bases. Determining relevant features leading to situations classification is the core of case-based reasoning. Hybrid systems mix reasoning methods e.g. ontological applications often combine DL and a FOL engine.

Several criteria led our choice for the engine core: semantic degrees, expressiveness (vs efficiency) and usability. We choose to base our representation on
CG. They have a strong semantic founding and are built on an ontology. They provide a good expressiveness (a universal knowledge representation (Sowa, 2008)(Chein and Mugnier, 2009)) equivalent to FOL but with a better usability since they are also human readable. The needed expressiveness is an open issue nevertheless You Can Only Learn What You Can Represent (Otterlo, 2009). Thus, it is a fundamental question for a sustainable use. FOL is usually the most expressive choice made for context-awareness. Meantime, semantic reasoning with an ontology is the most used approach in context-awareness as it provides interoperability and a non-abstract representation. Moreover coupled with the CG usability, the model may allow at some point a welcomed direct users involvement (Brézillon, 2011). Semantic virtual worlds as a new paradigm is a discussed issue (Latoschik et al., 2008). Several approaches offer frameworks to build full semantic worlds (Latoschik et al., 2005) (Lugrin and Cavazza, 2007). Lot of those works use semantic networks (Peters and Shrobe, 2003)(Lugrin and Cavazza, 2007)(Bonis et al., 2008) which reinforce our conviction for CG. However we will not try to build a full semantic world but to gather semantic information to help the 3D interaction. We aim at context-awareness in classic applications with an external representation and reasoning engine.

\section{Current work}

As shown in Figure 3, the engine manages context and decisions concerning the user, interaction and environment and communicates through different tools. Those tools must have a semantic description of their uses in order to be triggered by the engine. They can be actuators with visible effects or sensors that retrieve information. Those tools can embed other forms of reasoning than the engine core (e.g HMM) to provide information.

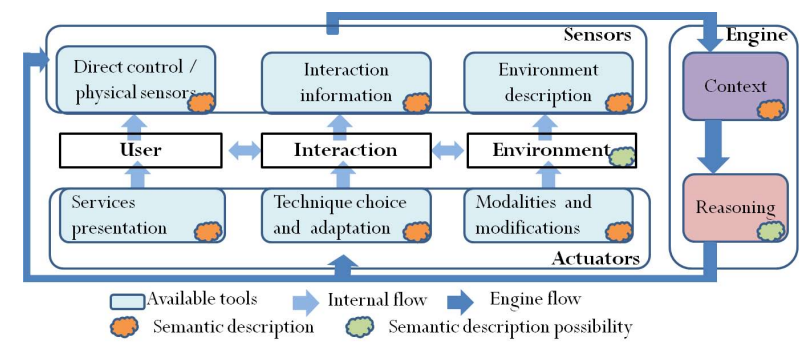

Figure 3: A parallel engine - communication though semantic tools

Context can have various forms as illustrated in Figure 4. First, the ontology lists concepts and rela- 
tions with underlying semantic, which are used by CG in order to describe rules and facts. Available tools and the past events in history are special facts. Events are newly integrated information and trigger a decision request in an automatic mode. The time manager role is to check the validity of the needed facts. When a decision with an associated tool is true, the engine aggregates its belief and risk from facts, events' timing and rules. The user cognitive load is represented by an acceptable total risk, which induces a knapsack problem as a last classification.

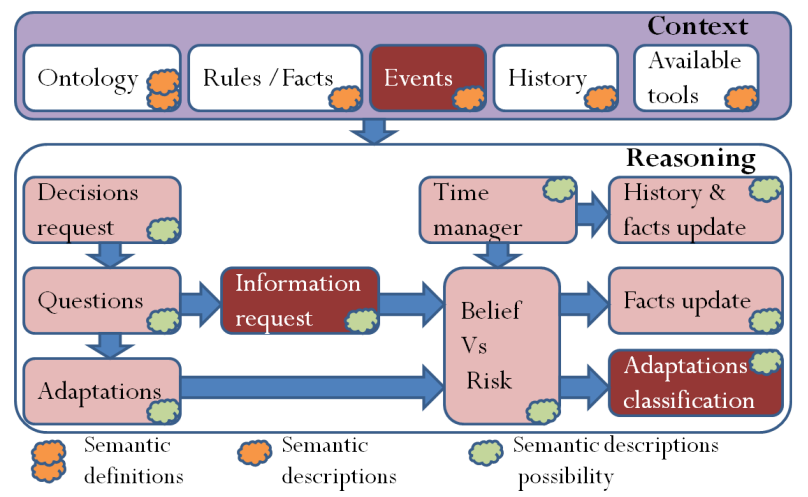

Figure 4: The engine - forms of context and reasoning

Context and reasoning blocks (Figure 4) can also be grouped by their role in the engine (Figure 5). The situation progresses with two roughly separated interlaced processes: decision and comprehension. The decision process goes from representing the situation to reasoning (KR \& R), and transforms simple facts beliefs into the best reaction bet. The comprehension process goes from identifying the situation to understanding how to assist (the two context-awareness trends) and transforms simple data to a full plan of the situation. The decision set is extended as the comprehension progresses (e.g an acquired interest can unlock a reactive adaptation)

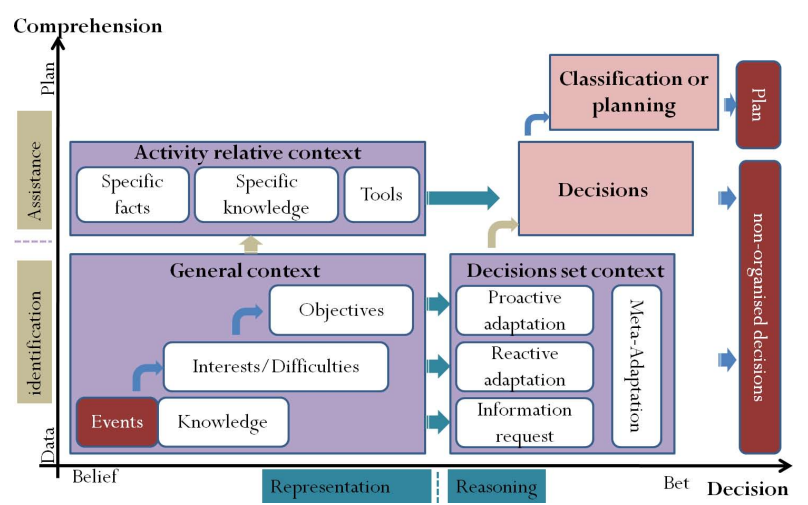

Figure 5: Hierarchy of concepts in the engine - Towards an assistance plan bet
We use Virtools as our scene graph manager and the Amine platform (Kabbaj, 2006) (a Java opensource multi-layer platform for intelligent systems) for the engine. This platform offers an ontology manager and a FOL engine that handles CG: Prolog+CG.

We are testing the engine on a small scenario. However, even simple cases pose several open questions: What items of information are pertinent? How to balance decisions risk and belief? How to best express a rule? How to monitor the user's intention?

The engine core combines general rules with scene tools and specific rules. We test the engine and those rules with a first case study: to try to automatically acquire some user's interests and enhance them. We first focus on interests linked to the user's hand. Thus the only specific rule is to monitor the "hand" object and to set a cognitive load. Then, several tools may help. We started by defining:

- a Zones Of Interest (ZOI) sensor that attaches auras to objects and reports their content;

- an object's movement sensor (movement is high/low, local/global);

- an actuator to change the color of an object;

- an actuator to add a haptic/visual gain to an object.

The engine's first reaction is to activate the sensors on the target "hand". Those sensors send events to the engine, e.g an event that describes an object inside the hand's ZOI. Events trigger a reaction request that finds adaptations with corresponding available tools: e.g both precedent actuators, with two uses for activation and deactivation. Engine's adaptation decisions use general rules like:

- Define interests (e.g in a ZOI);

- Try to enhance an interest;

- Associate possible enhancements in this situation: e.g object visual modifications through color change, as well as interaction modifications through gain (visual or haptic);

- Manage adaptations states :

- remove added visual modification if the object is not an interest;

- remove added gain if an object is an interest and the movement is abnormal (e.g local+high=the user is "stuck").

Next, the engine calculates the decisions belief and risk from the initial risk supplied by the tools and possibly beliefs for sensors events. Each rule has a belief transfer rate. This rate for a color change is greater than the one for a visual gain, as it is usually more suited with only the "enhancement will" context. Finally, other rules focuse on risk management:

- increase decision risk if some concepts are used (e.g haptic gain risk $>$ visual gain risk $>$ object visual modification risk); 


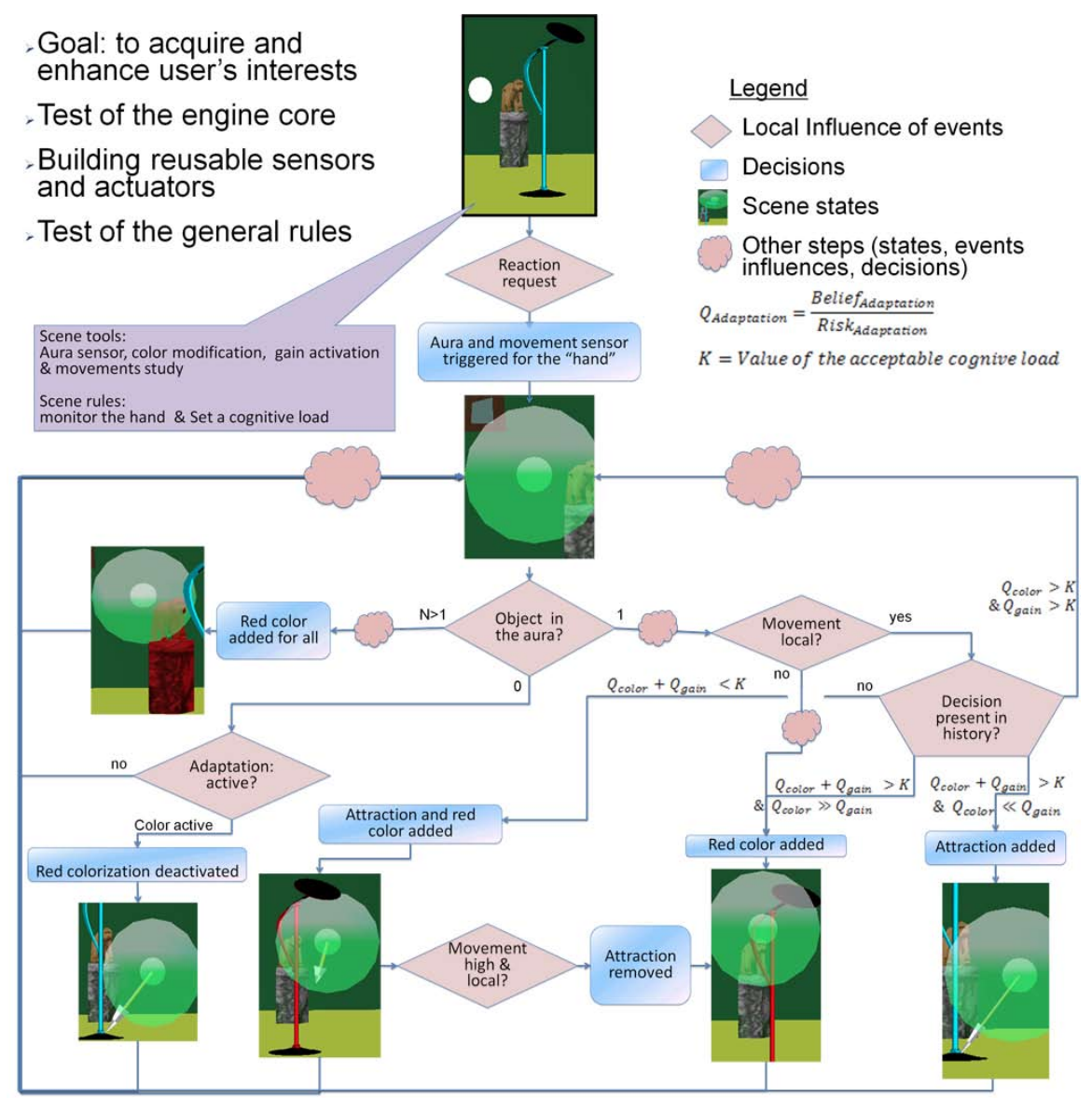

Figure 6: Case study - the engine automatically apply different adaptations depending on the context

- increase decision's risk if present in history (e.g avoid activation/deactivation cycle);

- decrease interaction modification's risk if movement is local.

As a result, the rules combine themselves as expected (Figure 6). Passing by an object makes its color red, while standing next to it makes it also attractive. Colors are reset when we move far away, while attraction is removed when we try to resist it. When it has been deactivated, gain cannot be reactivated for a time corresponding to history memory. Some reactivations can occur for coloring as the decision is initially less risky. Those results depend on the initial risk, belief and cognitive load values. More complex situations occur when several objects are close to the hand: e.g only the less risky adaptation is applied to a maximum of objects.

\section{Conclusion}

The setting up and implementation of an intelligent 3D interaction assistance and context man- ager with Amine platform and Conceptual Graphs is promising, and we are now improving it by reflections on examples. The engine aims to allow a semantic reasoning and the reuse of tools in a non-semantic environment to help the 3D interaction. We propose an engine core with a semantic base to achieve adaptation, which could be directly addressed by sensors, designers or users. Context and adaptations to be considered for virtual reality along with the user's degrees of appreciation and control are open questions which will guide the engine evolution. The significant engine prototype response delay is not suited for a full automatic mode yet, but rather for punctual helps. This drawback will be lessen but is an inherent part of our approach. Ultimately the engine core will be tested on more complex scenario. However, the next major step is to obtain enhanced user's intention hints.

\section{ACKNOWLEDGEMENTS}

This work is supported by the IBISC laboratory and the FP7 DigitalOcean project. 


\section{REFERENCES}

Aylett, R. and Luck, M. (2000). Applying artificial intelligence to virtual reality: Intelligent virtual environments. Applied Artificial Intelligence, 14(1):3-32.

Bettini, C., Brdiczka, O., Henricksen, K., Indulska, J., Nicklas, D., Ranganathan, A., and Riboni, D. (2010). Pervasive and Mobile Computing.

Bonis, B., Stamos, J., Vosinakis, S., Andreou, I., and Panayiotopoulos, T. (2008). A platform for virtual museums with personalized content. Multimedia Tools and Applications, 42(2):139-159.

Boudoin, P., Otmane, S., and Mallem, M. (2008). Fly Over, a 3D Interaction Technique for Navigation in Virtual Environments Independent from Tracking Devices. Virtual Reality, (Vric).

Bouyer, G., Bourdot, P., and Ammi, M. (2007). Supervision of Task-Oriented Multimodal Rendering for VR Applications. Communications.

Bowman, D. A., Chen, J., Wingrave, C. A., Lucas, J., Ray, A., Polys, N. F., Li, Q., Haciahmetoglu, Y., Kim, J.-s., Kim, S., Boehringer, R., and Ni, T. (2006). New Directions in 3D User Interfaces. International Journal,

Brézillon, P. (2011). From expert systems to context-based intelligent assistant systems : a testimony. Engineering, 26:19-24.

Celentano, A. and Nodari, M. (2004). Adaptive interaction in Web3D virtual worlds. Proceedings of the ninth international conference on $3 D$ Web technology - Web3D '04, 1(212):41.

Chein, M. and Mugnier, M. (2009). Graph-bases Knowledge Representation: Computational Foundations of Conceptual Graphs. Springer.

Coppola, P., Mea, V. D., Gaspero, L. D., Lomuscio, R., Mischis, D., Mizzaro, S., Nazzi, E., Scagnetto, I., and Vassena, L. (2009). AI Techniques in a Context-Aware Ubiquitous Environment, pages 150-180.

Dey, A. and Abowd, G. (2000). Towards a better understanding of context and context-awareness. In $\mathrm{CHI}$ 2000 workshop on the what, who, where, when, and how of context-awareness, volume 4.

Frees, S. (2010). Context-driven interaction in immersive virtual environments. Virtual Reality, Volume 14(4):1-14.

Irawati, S., Calderón, D., and Ko, H. (2005). Semantic 3D object manipulation using object ontology in multimodal interaction framework. In Proceedings of the 2005 international conference on Augmented teleexistence, pages 35-39. ACM.

Kabbaj, A. (2006). Development of Intelligent Systems and Multi-Agents Systems with Amine Platform. Intelligence, pages 1-14.

Latoschik, M. E., Biermann, P., and Wachsmuth, I. (2005). Knowledge in the Loop : Semantics Representation for Multimodal Simulative Environments. pages 25 39.

Latoschik, M. E., Blach, R., and Iao, F. (2008). Semantic Modelling for Virtual Worlds A Novel Paradigm for
Realtime Interactive Systems ? In ACM symposium on Virtual reality software and technology.

Lee, S., Lee, Y., Jang, S., and Woo, W. (2004). vr-UCAM: Unified context-aware application module for virtual reality. Conference on Artificial Reality.

Lugrin, J.-1. and Cavazza, M. (2007). Making Sense of Virtual Environments : Action Representation, Grounding and Common Sense. In 12th International conference on intelligent user interfaces.

Octavia, J., Coninx, K., and Raymaekers, C. (2010) Enhancing User Interaction in Virtual Environments through Adaptive Personalized 3D Interaction Techniques. In $U M A P$

Otterlo, M. (2009). The logic of adaptive behavior.

Paramythis, A. (2009). Adaptive Systems : Development, Evaluation and Evolution. $\mathrm{PhD}$ thesis.

Peters, S. and Shrobe, H. E. (2003). Using Semantic Networks for Knowledge Representation in an Intelligent Environment. In 1st International Conference on Pervasive Computing and Communications.

Poupyrev, I., Billinghurst, M., Weghorst, S., and Ichikawa T. (1996). The go-go interaction technique: non-linear mapping for direct manipulation in VR. In Proceedings of the 9th annual ACM symposium on User interface software and technology, pages 79-80. ACM.

Preuveneers, D. (2009). Support for context-driven applications in Ambient Intelligence environments.

Soto, M., Allongue, S., Lip, L., Place, C., Cedex, P., Soto, M., and Allongue, S. (1997). A Semantic Approach of Virtual Worlds Interoperability. In Proceedings Sixth IEEE workshops on Enabling Technologies: Infrastructure for Collaborative Enterprises.

Sowa, J. F. (2008). Conceptual Graphs, pages 213-217. Elsevier.

Wingrave, C. A., Bowman, D. A., and Ramakrishnan, N. (2002). Towards Preferences in Virtual Environment Interfaces. Interfaces. 